\title{
Moving target localization in indoor wireless sensor networks mixed with LOS/NLOS situations
}

\author{
Yunzhou Zhang ${ }^{*}$, Wenyan Fu', Dongfei Wei, Jianjun Jiang and Bing Yang
}

\begin{abstract}
Indoor target localization is an essential and fundamental issue for wireless sensor networks (WSN). However, it is rather difficult for WSN to maintain the localization accuracy in line-of-sight (LOS) and non-line-of-sight (NLOS) mixed environment. NLOS propagation always leads to larger ranging error than LOS does. When the target moves in the rooms and corridors, the signal transmission state will switch frequently between LOS and NLOS. It is a challenging task to deal with this situation because the ranging error characteristics under LOS and NLOS conditions are quite different. In this paper, we propose an interacting multiple model-extended Kalman filter (IMM-EKF) algorithm to improve the localization accuracy for moving target in indoor environment. In the IMM structure, two Kalman filters (KF) are adopted in parallel to accurately smoothen the distance measurement. The proposed algorithm can adapt to the dynamically changing condition between LOS and NLOS due to the two KFs' interaction so that large NLOS ranging errors are further reduced. Once the estimated ranges are obtained, the EKF is employed to estimate the target's location. Empirical measurement results are obtained from typical office environment to verify the effectiveness of the proposed algorithm. Experimental results illustrate that the IMM smoother can efficiently mitigate the NLOS effects on ranging errors and achieve high localization accuracy.
\end{abstract}

Keywords: Moving target localization; Wireless sensor networks; Indoor environment; LOS/NLOS; Interacting multiple model

\section{Introduction}

In recent years, indoor target localization and tracking technologies are undoubtedly booming in various fields such as robotics and emergency systems. GPS localization is not suitable for indoor environment due to the building's occlusion [1]. Since it owns many advantages such as reliable data and high positioning accuracy, wireless sensor networks (WSN) is widely used for indoor target localization.

According to the relative position between anchor node (AN) and target node (TN), the propagation condition of wireless signal can be divided into such two situations as line-of-sight (LOS) and non-line-of-sight (NLOS). As shown in Figure 1, LOS link represents the direct signal propagation between $\mathrm{AN}$ and TN without obstruction. However, in WSN, especially in indoor environment, due to the obstruction such as building

\footnotetext{
* Correspondence: zhangyunzhou@ise.neu.edu.cn

${ }^{\dagger}$ Equal contributors

College of Information Science and Engineering, Northeastern University, WenHua Road, HePing District, Shenyang 110819, China
}

structure, furniture, and human activity [2-4], the signal propagation will fall into reflection, diffraction, scattering, etc. This indirect signal propagation is defined as NLOS. Whether measured by time-of-arrival (TOA) or received signal strength (RSS), the distances obtained under NLOS link always contain obvious positive bias because the signal travels longer path or time than direct link. Furthermore, the measurement error under NLOS is often larger than that under LOS. With chirp spread spectrum (CSS) nodes $[5,6]$, the different measurement noises under LOS and NLOS condition are shown in Figure 2. Generally, a mixture of LOS and biased NLOS measurements will lead to large error, which is a significant challenge in practice. This paper focuses on how to maintain the accuracy of the mobile localization in indoor environment mixed with LOS/NLOS conditions.

Several techniques [7-12] have been proposed to mitigate the bad effect of NLOS measurement error. The basic approach is to identify the NLOS condition and then remove associated measurements $[7,8]$. In this way, the adverse effect of NLOS propagation is eliminated 


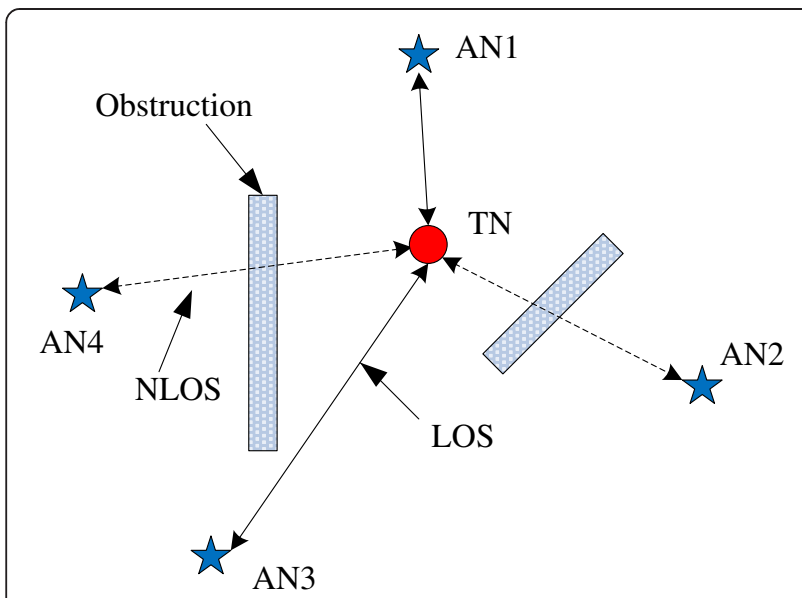

Figure 1 Signal propagation under LOS and NLOS.

and traditional localization methods can be used to obtain accurate positions. Constrained optimization techniques have also been applied to reduce NLOS errors $[9,10]$. Furthermore, some researchers even try to explore new methods without identification of LOS/NLOS, but these methods always need either abundant prior knowledge [11] or complex training process [12].

Indoor environment is very complex and should be considered carefully due to the presence of obstructions such as walls, doors, and corridors. When the moving target $(\mathrm{MN})$ moves through rooms and corridors, the propagation conditions change between LOS and NLOS alternatively over a short distance. The range estimation conducted with the NA5TR1 CSS nodes shows that the ranging error characteristics are quite different between LOS and NLOS measurements. This frequent LOS/ NLOS transition will cause a serious measurement error. Therefore, it is significant to cope with the dynamically

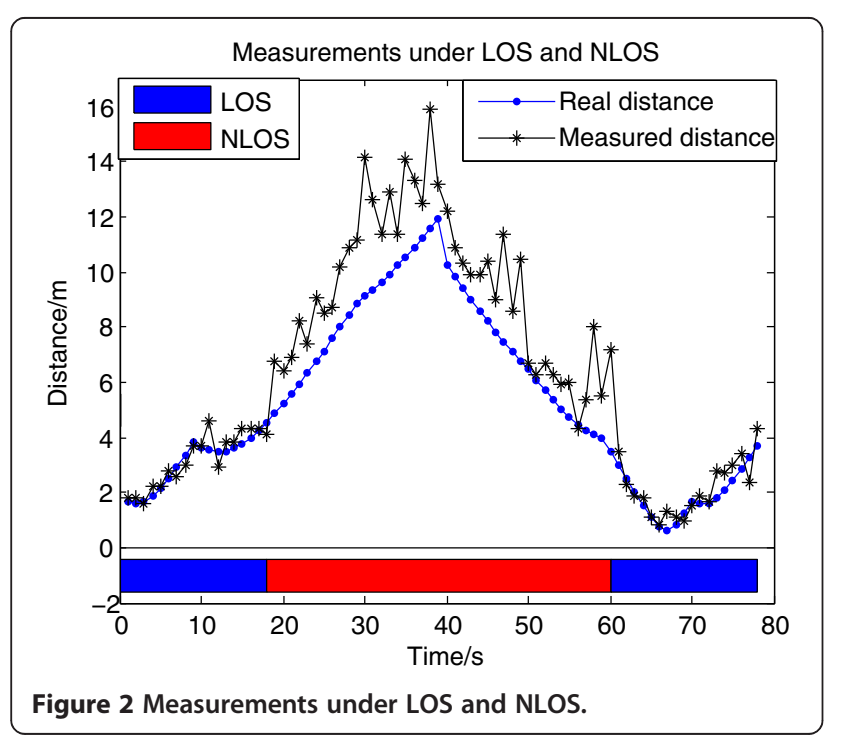

changing propagation for mobile localization in indoor environment mixed with LOS and NLOS situations. For this case, the transmission channel between the MN and ANs can be treated as a switching mode. We need to determine the LOS/NLOS probabilities of the propagation path between the MN and each AN, respectively. Some proper strategies, such as first-order Markov [13] and IMM $[14,15]$, were proposed to deal with this switching mode system. IMM estimator has been demonstrated as one of the most effective methods to estimate the state of a hybrid dynamic system with several switching modes under uncertain conditions.

In this paper, we focus on how to achieve high accuracy under the dynamic changing LOS/NLOS conditions in indoor environment. We use a Kalman filtering-based interacting multiple model (KF-IMM) smoother to filter the distance measurement in indoor LOS/NLOS situations. Through the LOS and NLOS error analysis on off-line measurements, we get the error parameters in two propagation conditions. After that, we respectively set up two channel error parameters of the Kalman filter model for IMM structure. These two KF filters smooth the distance measurements at the same time, and the model probabilities are calculated adaptively based on the error parameters. Then, the measurement error can be mitigated at the output combination stage. As the filtered distances are obtained, the EKF is employed to achieve the target position. To validate the performance of the proposed method, we conducted the experiment on the fifth floor of the Information Building at Northeastern University.

The main contributions of this paper are listed as follows:

(1) We propose a new idea to deal with the frequent transition between NLOS and LOS conditions. Based on the ranging information, the proposed IMM filtering algorithm can adaptively calculate the probabilities of LOS model and NLOS model. Then, the two models interact through the Markov chain automatically. Therefore, the range smoother can response in time when the LOS/NLOS conditions changes. This is significant for range filtering in LOS/NLOS mixed environment.

(2)By our proposed algorithm, the filtered results of LOS and NLOS models can be combined automatically by IMM structure. According to the different error characteristics of LOS and NLOS, we adopt two parallel KFs. The range filtering results of the two KFs are combined based on the model probabilities. In this way, it is not only feasible for severe NLOS condition, but also viable to various degrees of NLOS.

(3) We conduct extensive experiments to validate the analysis and evaluate the performance of proposed 
algorithm. Experimental results show that positive NLOS error is significantly mitigated and moving target localization can be accurately achieved.

The remainder of this paper is organized as follows: Section 2 gives a brief overview of the localization techniques in NLOS environments. The system model is described in Section 3. In Section 4, the IMM smoother and EKF-based localization algorithm are proposed. The performance of our algorithm in practical indoor environment is presented in Section 5. Finally, the conclusion is drawn in Section 6.

\section{Related works}

Traditional localization algorithm based on WSN mainly includes trilateration localization, triangulation localization, and least squares. These algorithms directly measure the ranges or angles, which are used for the localization. Under the LOS environment, these algorithms can achieve high accuracy. But for NLOS environment, they cannot obtain desired accuracy because of the significant NLOS measurement error $[16,17]$.

Nowadays, researches toward target localization in NLOS environments mainly focus on LOS/NLOS identification and NLOS error mitigation. The basic idea of NLOS identification algorithm can be divided into two types, including measurement-based identification $[18,19]$ and the channel statistics-based identification [20-22]. An angle of arrival (AOA)-based NLOS identification for multiple-antenna system is proposed in [18]. On the basis of the phase difference across two antenna elements, the hypothesis test on $\mathrm{K}$-factor is adopted to identify the NLOS state. However, the limitation lies in that AOA-based measurement needs additional hardware support. Reference [19] presents a TOA-based NLOS identification method with prior knowledge such as the standard deviation of measurement noise. RSS-based identification algorithms are also widely used, but the ideal accuracy can only be achieved in ultra wideband condition [20]. In [21], the statistics of radio propagation channel metrics along with binary hypothesis testing are applied to identify the NLOS states. In [22], the RSS and time characteristic are utilized to estimate the NLOS situations, but the analysis process is relatively complicated.

Most of the NLOS mitigation technologies are dependent upon the NLOS identification. Residual test algorithm, presented in [23], can identify LOS ANs to avoid NLOS impact by discarding the NLOS measurement. However, it might be unfeasible when the number of available range measurements is limited. In [11] and [24], a non-parametric approach is proposed for NLOS identification based on support vector machines, which can attain good accuracy at the cost of high computation. In [25], a linear programming (LP) approach is adopted for NLOS mitigation. Some researchers propose soft-decision algorithms without NLOS identification and discarding [26-28]. In [26,27], Chen uses a residual weighting (RWgh)-based algorithm to alleviate the NLOS errors. On the basis of Chen's research, Hammes and Zoubir [28] suggest a data fusion algorithm to achieve higher localization accuracy. However, RWgh algorithms cannot perform well when the number of LOS nodes is deficient. But, the computational complexity will grow rapidly when the number of ANs increases. In [29], vehicle velocity and heading direction measurements are exploited to make constrained optimization for NLOS error mitigation. Other researchers propose some model matching and database linked methods, such as fingerprinting algorithm, which are always time-consuming [12]. Some researchers begin to explore new methods without NLOS identification. Typical algorithms include least square multi-lateration [30], optimized residual weighting [31], support vector machine classifier [11], and machine learning [32]. These methods often require ideal synchronization or massive experimental data.

As for frequent LOS/NLOS transition, researchers have made good progress with interacting multiple mode. Yang et al. [33] propose a location estimation scheme using fuzzy-based IMM smoother for mobile location. IMM is employed as a switch between the LOS and NLOS states, which are considered to be a Markov process with two interactive modes. Associated with data fusion, this scheme can efficiently mitigate the NLOS effects on the measurement. In [15], interacting multiple model is proposed for moving target location estimation in cellular network. In the rough wireless environment such as urban area, the proposed method can efficiently mitigate the NLOS effects on the ranging error. However, the urban structure is quite different from indoor environment. The NLOS situation in urban area is caused by obstruction due to large buildings, while the indoor NLOS always considers the signal attenuated by walls or doors. Taking this into consideration, the measurement model should be different between these two environments.

\section{System model}

The WSN-based indoor moving target localization system contains several ANs and a moving node (MN). MN communicates with ANs to acquire distance measurements, with which the position of $\mathrm{MN}$ at a particular time step can be estimated.

At the time step $t$, the distances obtained through the communication between MN and M ANs by CSS system are used to build the distance vector:

$$
D_{A r r}^{\text {mes }}(t)=\left[d_{1}^{\text {mes }}(t), d_{2}^{\text {mes }}(t), \cdots, d_{M}^{\text {mes }}(t)\right]^{\mathrm{T}},
$$


where $d_{m}^{\text {mes }}(t)$ is the range measurement between the $m$ th $\mathrm{AN}$ and the MN. Our experiment is operated in the office building as shown in Figure 3. The channel conditions between $\mathrm{MN}$ and ANs change dynamically when the target moves. The statistics of channel conditions between the moving $\mathrm{MN}$ and ANs are shown in Figure 4. The distances between $\mathrm{AN}$ and $\mathrm{MN}$ are directly acquired by NA5TR1 CSS node ranging, which is defined in the standard IEEE 802.15.4a [5,6]. CSS ranging is based on the time of flight (TOF) of radio frequency signals. In the CSS system, the TOF is calculated by symmetric double-sided two-way ranging (SDS-TWR) [34]. In this paper, we assume that the error parameters, which are calculated with offline measurements, of LOS and NLOS follow the normal distribution.

At time step $t$, the range state vector between the $m$ th $\mathrm{AN}$ and the $\mathrm{MN}$ is defined as

$$
D_{m}(t)=\left[d_{m}(t), \dot{d}_{m}(t)\right]^{\mathrm{T}} \quad m=1,2, \cdots, M
$$

where $d_{m}(t)$ represents the distances between the $m$ th AN and the MN, $\dot{d}_{m}(t)$ is the velocity of the MN according to $m$ th $\mathrm{AN}$, and $M$ is the number of the ANs. The state-space model can be expressed as linear dynamic equations

$$
D_{m}(t)=F D_{m}(t-1)+B \omega_{d}(t-1)
$$

where

$$
F=\left[\begin{array}{cc}
1 & T i \\
0 & 1
\end{array}\right], \quad B=\left[\begin{array}{c}
T i^{2} / 2 \\
T i
\end{array}\right] .
$$

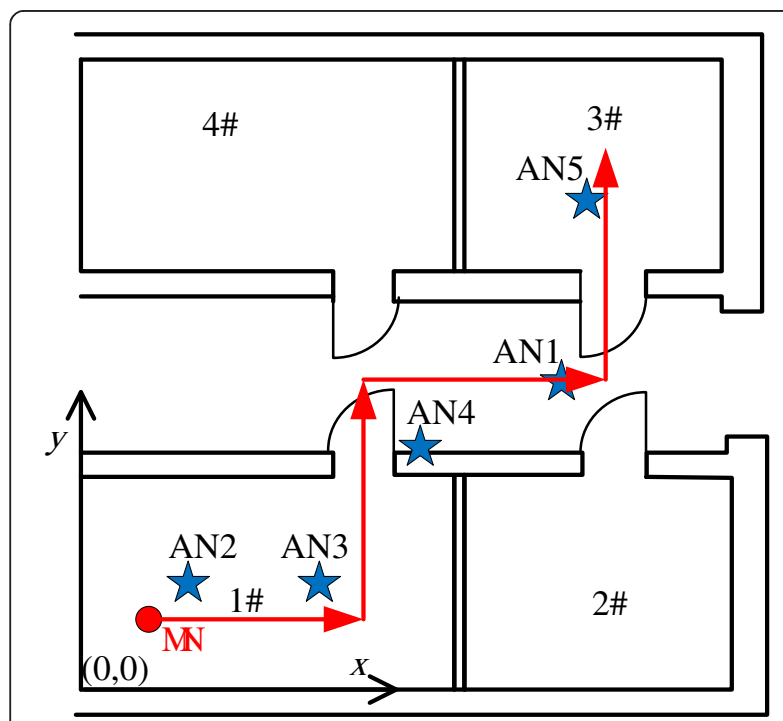

Figure 3 Deployment of the environment.

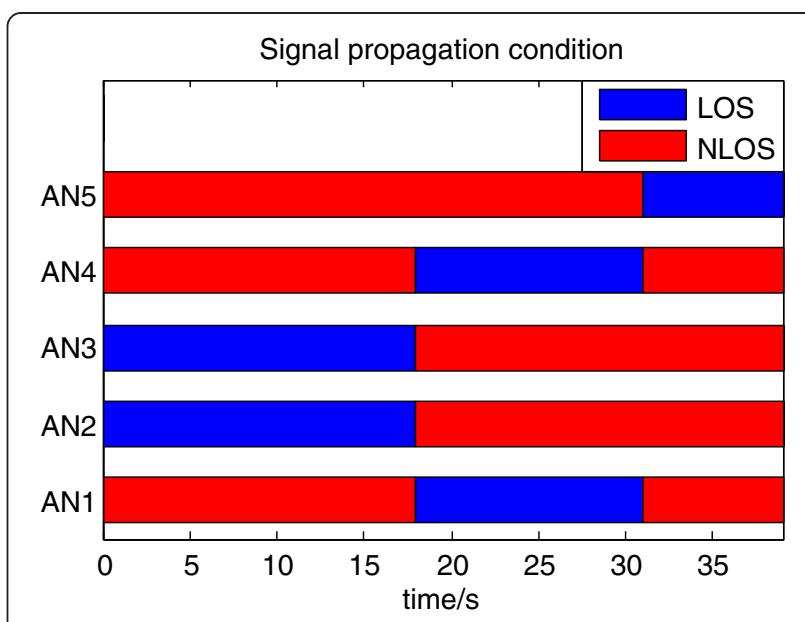

Figure 4 LOS/NLOS condition statistics.

$T i$ represents the sampling time interval, $\omega_{d}(t-1)$ denotes the process noise, which is assumed as independently and identically distributed (i.i.d.) Gaussian noise, with zero mean and covariance matrix $Q_{\mathrm{d}}$.

The MN's state vector at each time step $t$ is defined as a two-dimensional position and velocity vector of the $\mathrm{MN}$.

$$
X(t)=[x(t), \dot{x}(t), y(t), \dot{y}(t)]^{\mathrm{T}}
$$

where $(x(t), y(t))$ denotes the coordinate of the MN at time step $t$, while $(\dot{x}(t), \dot{y}(t))$ represents the velocity of the $\mathrm{MN}$ in $x$ and $y$ directions. The MN's state updates over time according to the random force model.

$$
X(t)=A X(t-1)+C \omega(t-1) \quad t=1,2, \cdots, T,
$$

where

$$
A=\left[\begin{array}{cccc}
1 & T i & 0 & 0 \\
0 & 1 & 0 & 0 \\
0 & 0 & 1 & T i \\
0 & 0 & 0 & 1
\end{array}\right], \quad C=\left[\begin{array}{cc}
T i^{2} / 2 & 0 \\
0 & T i^{2} / 2 \\
T i & 0 \\
0 & T i
\end{array}\right]
$$

$X(t-1)$ represents the posterior state at time step $t-1$. The process noise $\omega(t-1)$ is modeled as zero mean, i.i.d. Gaussian noise with covariance matrix $Q$.

The range measurement matrix has been defined in Equation (1). Then, let

$$
D_{\text {Arr }}^{\text {mes }}(t)=h(X(t))+v(t) \quad t=1,2, \cdots, T,
$$

where $h(X(t))=\left[h_{1}(X(t)), h_{2}(X(t)), \cdots, h_{M}(X(t))\right]^{\mathrm{T}}$ describes the Euclidean distance between $\mathrm{MN}$ and the ANs; the subelements are defined as

$$
\begin{aligned}
h_{m}(X(t)) & =\sqrt{\left(x_{m}-x(t)\right)^{2}+\left(y_{m}-y(t)\right)^{2}} \\
m & =1,2, \cdots, M,
\end{aligned}
$$

$v(t)=\left[v_{1}(t), v_{2}(t), \cdots, v_{M}(t)\right]^{\mathrm{T}}$ represents the range measurement noises, which are random variables with mean 
error $\mu_{d}$ and variance $\sigma_{d}^{2}$. Here, $\mu_{d}$ and $\sigma_{d}^{2}$ are assumed to be i.i.d. Gaussian with different parameters according to the LOS and NLOS states

$$
\begin{aligned}
\mu_{d} & =\left\{\begin{array}{cc}
0, & \text { LOS } \\
\mu_{d}^{\text {nlos }}, & \text { NLOS }
\end{array}\right. \\
\sigma_{d}^{2} & =\left\{\begin{array}{cc}
R^{\text {los }}, & \text { LOS } \\
R^{\text {nlos }}, & \text { NLOS }
\end{array}\right.
\end{aligned}
$$

\section{Target localization based on IMM algorithm}

Since the indoor construction is complicated and the communication signals are obstructed frequently, the NLOS propagation condition will be caused between $\mathrm{MN}$ and ANs. However, the measurement errors under LOS and NLOS conditions are quite different. Since the uniform measurement filtering method cannot fit both LOS and NLOS conditions, it is necessary to introduce a mixture of LOS and biased NLOS range filter. Therefore, we adopt the IMM algorithm to filter the measurements under the LOS/NLOS conditions. Then, EKF is employed to estimate the MN's location.

\subsection{Measurement filtering based on IMM algorithm}

Single model is insufficient to capture the measurements under LOS/NLOS mixed environment, because the measurement errors are quite different between LOS and NLOS conditions. The IMM structure employs two parallel Kalman filters to smoothen the measurement errors in LOS and NLOS separately. Then, the state estimates of both filters are combined automatically according to the model probabilities. As the MN moves, due to the construction and furniture, the communication conditions between $\mathrm{MN}$ and ANs frequently alternate between LOS and NLOS. The LOS/NLOS transition channel between $\mathrm{AN}$ and $\mathrm{MN}$ is considered as a switching-mode system, which can be seen as a firstorder time-homogeneous Markov chain [15] depicted in Figure 5. Markov chain consists of two states. We let $i$ and $j(i, j=1,2)$ denote the model variable, where $i, j=1$ represents the LOS model, and $i, j=2$ represents the NLOS model. The transition probabilities $p_{i j}$ denote the conditional probability of states transition from status $i$ at time step $t-1$ to status $j$ at time step $t$. Let $D_{i}(t)$ denote the input of the state model $i$ at the time step $t$, then

$$
\begin{aligned}
& D_{1}(t)=p_{11}^{\prime} \cdot D_{1}(t-1)+p_{21}^{\prime} \cdot D_{2}(t-1) \\
& D_{2}(t)=p_{12}^{\prime} \cdot D_{1}(t-1)+p_{22}^{\prime} \cdot D_{2}(t-1),
\end{aligned}
$$

where

$$
\begin{array}{ll}
p_{11}^{\prime}=\frac{p_{11}}{p_{11}+p_{21}}, & p_{21}^{\prime}=\frac{p_{21}}{p_{11}+p_{21}}, \\
p_{12}^{\prime}=\frac{p_{12}}{p_{12}+p_{22}}, & p_{22}^{\prime}=\frac{p_{22}}{p_{12}+p_{22}}
\end{array}
$$

represent the normalized transition probabilities, and $p_{11}+p_{12}=1$.

The framework of the IMM algorithm is illustrated in Figure 6. The IMM algorithm consists of four steps: input interaction, model filtering, model probability update, and output combination.

\subsubsection{Input interaction}

Since the IMM algorithm is recursive, the inputs of both KFs in the IMM structure at time step $t$ come from the two KFs' filtering results at time step $t-1$. The mixing probability $\mu_{m, i \mid j}(t \mid t-1)$ representing the mixing probability of the $i$ th KF filtering result inputs to $j$ th KF filter can be calculated as

$$
\mu_{m, i l j}(t \mid t-1)=\left(1 / \bar{c}_{m, j}\right) p_{i j} u_{m, i}(t-1)
$$

where $\mu_{m, i}(t-1)$ represents the $i$ th model probability of the $m$ th AN at time step $t-1, p_{i j}$ denotes the transition probability defined in Markov chain, and $\bar{c}_{m, j}$ is a normalized variable, which is defined as

$$
\bar{c}_{m, j}=\sum_{i=1}^{2} p_{i j} u_{m, i}(t-1) .
$$

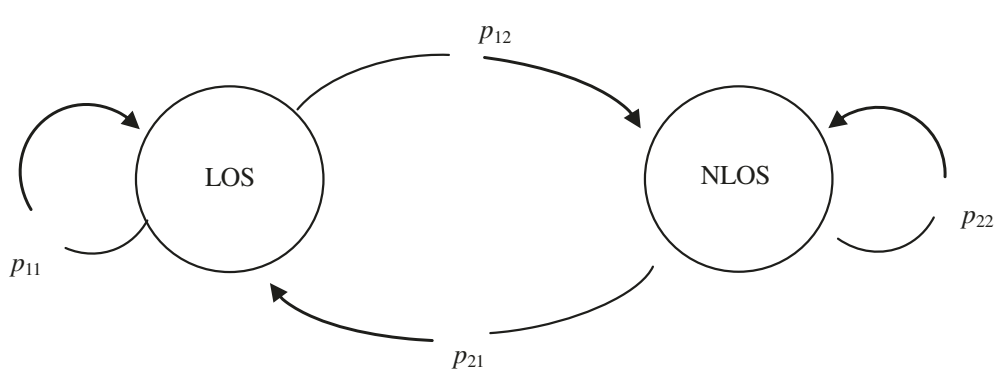

Figure 5 Markov chain. 


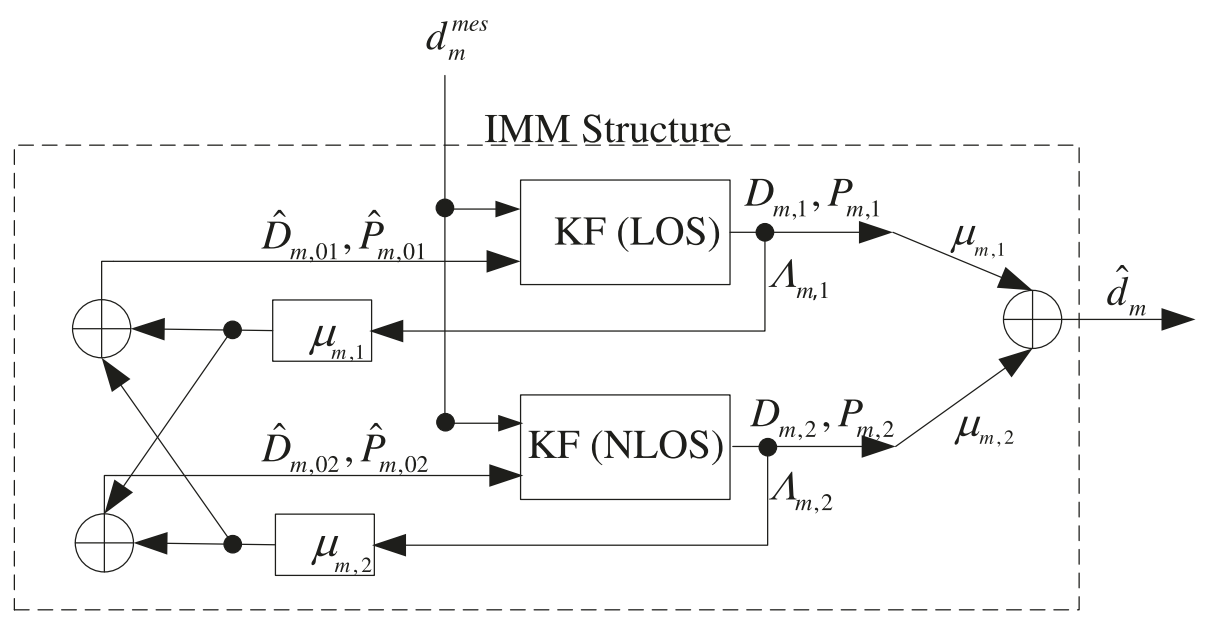

Figure 6 Structure of IMM.

With the input mixing probabilities, the state estimation for mode input $\hat{D}_{m, 0 j}(t \mid t-1)$ and a priori covariance matrix for the $j$ th mode-matched filter of $m$ th AN can be computed as

$$
\begin{aligned}
\hat{D}_{m, 0 j}(t \mid t-1)= & \sum_{i=1}^{2} \hat{D}_{m, i}(t-1 \mid t-1) u_{m, i l j}(t \mid t-1) \\
\hat{P}_{m, 0 j}(t \mid t-1)= & \sum_{i=1}^{2}\left\{P_{m, i}(t-1 \mid t-1)\left[D_{m, i}(t-1 \mid t-1)-\hat{D}_{m, 0 j}(t \mid t-1)\right]\right. \\
& {\left.\left[D_{m, i}(t-1 \mid t-1)-\hat{D}_{m, 0 j}(t \mid t-1)\right]^{\mathrm{T}}\right\} \mu_{m, i j}(t \mid t-1), }
\end{aligned}
$$

where $P_{m, i}(t-1 \mid t-1)$ denotes $i$ th model's posterior probability at time step $t-1$.

\subsubsection{Model filtering}

As mentioned above, we adopt two parallel KFs in this step. Based on the a priori knowledge that the measurement noises are quite different between LOS and NLOS conditions, one KF is designed for LOS measurement filtering and another is for NLOS. The KF can be described in two steps as

Prediction:

$$
\begin{aligned}
& \hat{D}_{m, j}(t \mid t-1)=F \hat{D}_{m, 0 j}(t \mid t-1) \\
& \hat{P}_{m, j}(t \mid t-1)=F \hat{P}_{m, 0 j}(t \mid t-1) F^{\mathrm{T}}+B Q_{d} B^{\mathrm{T}} .
\end{aligned}
$$

Update:

$$
K_{m, j}(t)=\hat{P}_{m, j}(t \mid t-1) G^{\mathrm{T}}\left[G \hat{P}_{m, j}(t \mid t-1) G^{\mathrm{T}}+G \sigma_{d} G^{\mathrm{T}}\right]^{-1}
$$

$$
V_{m, j}(t)=d_{m}^{m e s}(t)-G \hat{D}_{m, j}(t \mid t-1)
$$

$$
\begin{aligned}
& D_{m, j}(t \mid t)=\hat{D}_{m, j}(t \mid t-1)+K_{m, j}(t) V_{m, j}(t) \\
& P_{m, j}(t \mid t)=\left[I-K_{m, j}(t) G\right] \hat{P}_{m, j}(t \mid t-1),
\end{aligned}
$$

where $G=[1,0], I=[1,0 ; 0,1]$.

\subsubsection{Model probability update}

When the estimated states are obtained, the model likelihoods and probabilities are needed to be calculated to prepare for the output combination.

$$
\Lambda_{m, j}(t)=N\left(V_{m, j}(t) \mid 0, S_{m, j}(t)\right),
$$

where $\Lambda_{m, j}(t)$ denotes the Gaussian density function of residual error $V_{m, j}(t)$, with zero mean and covariance $S_{m, j}(t)$.

$$
\begin{aligned}
& S_{m, j}(t)=G P_{m, j}(t \mid t-1) G^{\mathrm{T}} \\
& u_{m, j}(t)=\frac{1}{c_{m, j}} \Lambda_{m, j}(t) \bar{c}_{m, j} \\
& c_{m, j}=\sum_{i=1}^{2} \Lambda_{m, i}(t) \bar{c}_{m, i} .
\end{aligned}
$$

\subsubsection{Output combination}

According to the model probability, the estimated distance can be updated as

$$
D_{m}(t \mid t)=\sum_{i=1}^{2}\left(D_{m, i}(t \mid t)-\mu_{d, i}\right) u_{m, i}(t)
$$




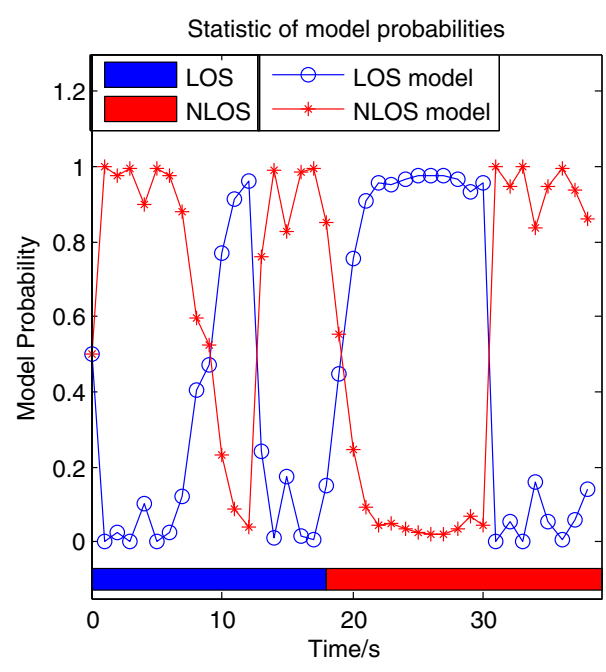

a

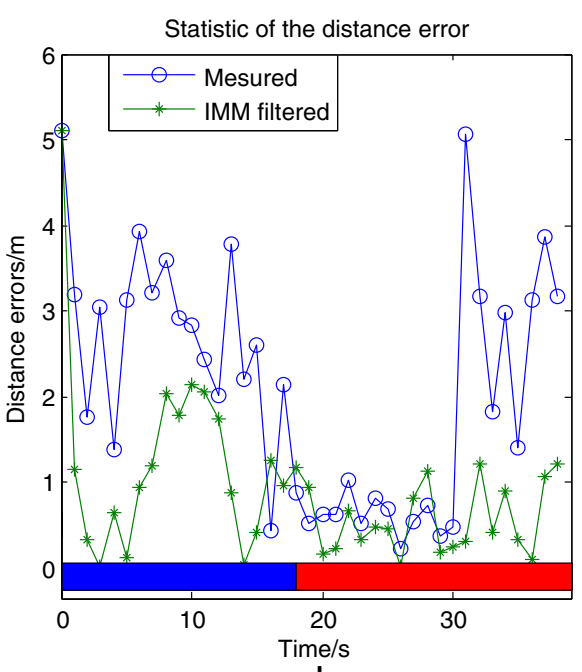

b

Figure 7 The performance of the IMM filter. (a) Model probabilities. (b) Distance errors along the path.

The value of $\mu_{d, i}$ refers to Equation (9), $i=1$ means LOS condition, $i=2$ means NLOS condition.

$$
\begin{aligned}
P_{m}(t \mid t)= & \sum_{i=1}^{2}\left\{P_{m, i}(t \mid t)+\left[D_{m, i}(t \mid t)\right.\right. \\
& \left.\left.-D_{m}(t \mid t)\right]\left[D_{m, i}(t \mid t)-D_{m}(t \mid t)\right]^{\mathrm{T}}\right\} u_{m, i}(t) .
\end{aligned}
$$

The final distance estimation can be calculated as

$$
\hat{d}_{m}(t)=G D_{m}(t \mid t) .
$$

\subsection{Mobile localization}

We use EKF to deal with non-linear dynamic problems of the MN localization. The basic idea of EKF is to convert the non-linear system according to the one-order Taylor function expansion into the linear system.

The process of EKF-based MN localization is described as

Prediction:

$$
\begin{aligned}
& \hat{X}(t \mid t-1)=A X(t-1 \mid t-1) \\
& \hat{P}(t \mid t-1)=A P(t \mid t-1) A^{\mathrm{T}}+C Q C^{\mathrm{T}} .
\end{aligned}
$$

Update:

$$
Z(t)=h(X(t \mid t-1))
$$

Table 1 Statistics of distance error

\begin{tabular}{llllll}
\hline Mean error/m & AN1 & AN2 & AN3 & AN4 & AN5 \\
\hline Measurement & 2.11 & 1.04 & 1.33 & 1.22 & 1.87 \\
IMM filter & 0.84 & 0.83 & 0.58 & 0.61 & 0.80 \\
\hline
\end{tabular}

$$
V(t)=\hat{D}_{A r r}(t)-Z(t)
$$

where $\hat{D}_{A r r}(t)=\left[\hat{d}_{1}(t), \hat{d}_{2}(t), \cdots, \hat{d}_{M}(t)\right]^{\mathrm{T}}$ denotes the estimated measurement vector obtained from the IMM filtering. Then, the Jacobian matrix $H$ can be defined as

$$
\begin{aligned}
& H=\left.\frac{\partial h\left([x, y]^{\mathrm{T}}\right)}{\partial[x, y]^{\mathrm{T}}}\right|_{x=\hat{x}(t \mid t-1), y=\hat{y}(t \mid t-1)} \\
& K(t)=\hat{P}(t \mid t-1) H^{\mathrm{T}}\left[H \hat{P}(t \mid t-1) H^{\mathrm{T}}+H R H^{\mathrm{T}}\right]^{-1} \\
& X(t \mid t)=\hat{X}(t \mid t-1)+K(t) V(t)
\end{aligned}
$$

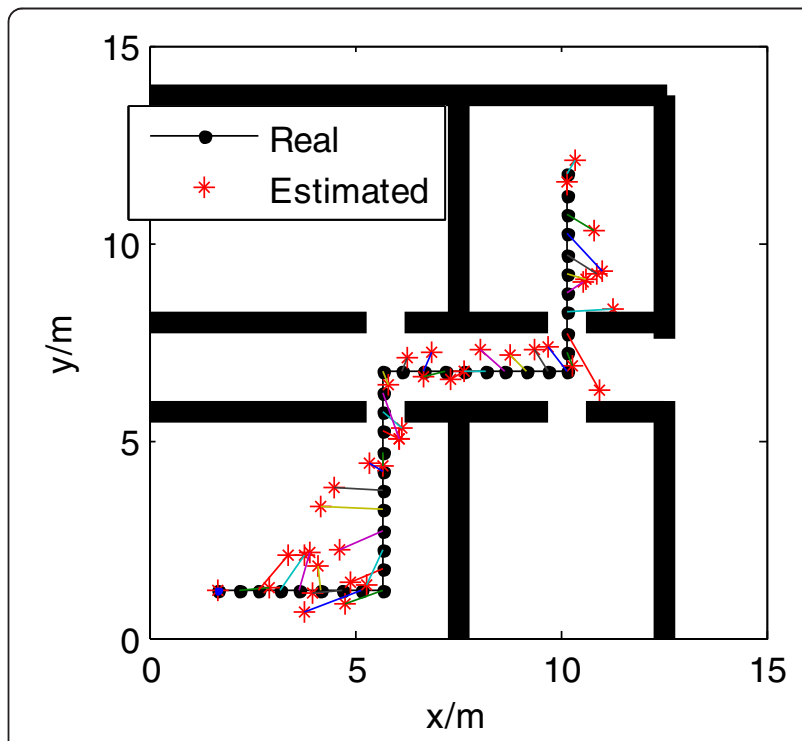

Figure 8 IMM-EKF tracking result. 


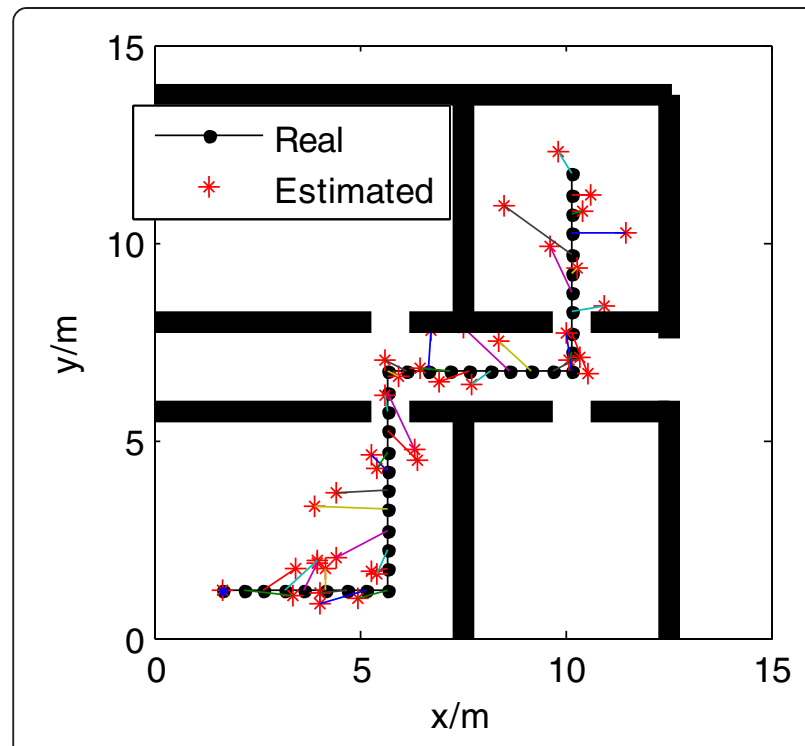

Figure 9 IMM tracking result.

$$
P(t \mid t)=[I-K(t) H] \hat{P}(t \mid t-1)
$$

The measured distances from LOS or NLOS have been mitigated by IMM. Furthermore, the EKF has smoothed the estimated error at the localization stage. Combining the IMM and EKF, we can get a more accurate estimation.

\section{Experimental result and analysis}

To evaluate the performance of our proposed algorithm, practical experiments are performed on the fifth floor of the Information Building of Northeastern

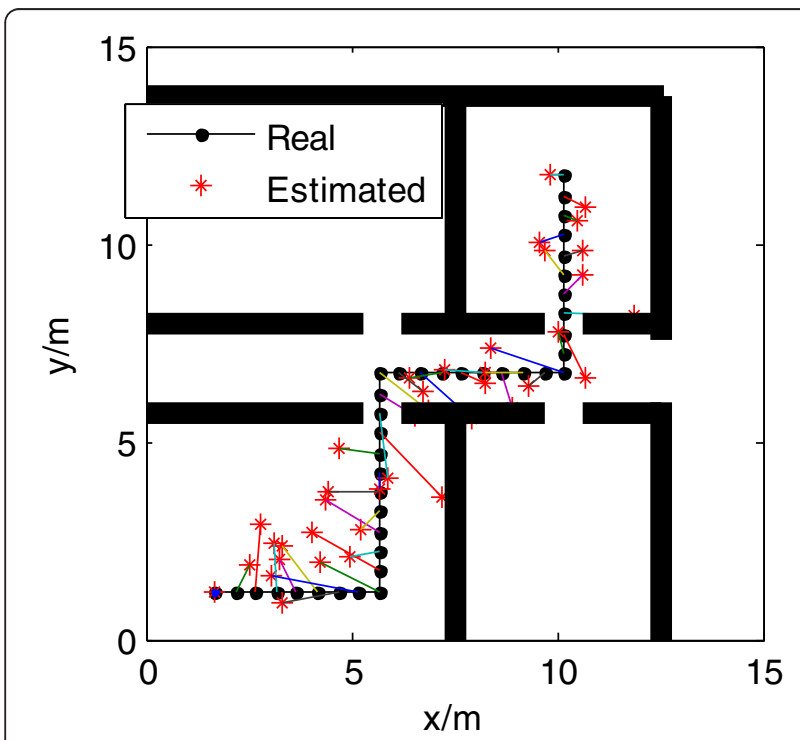

Figure 10 EKF tracking result.

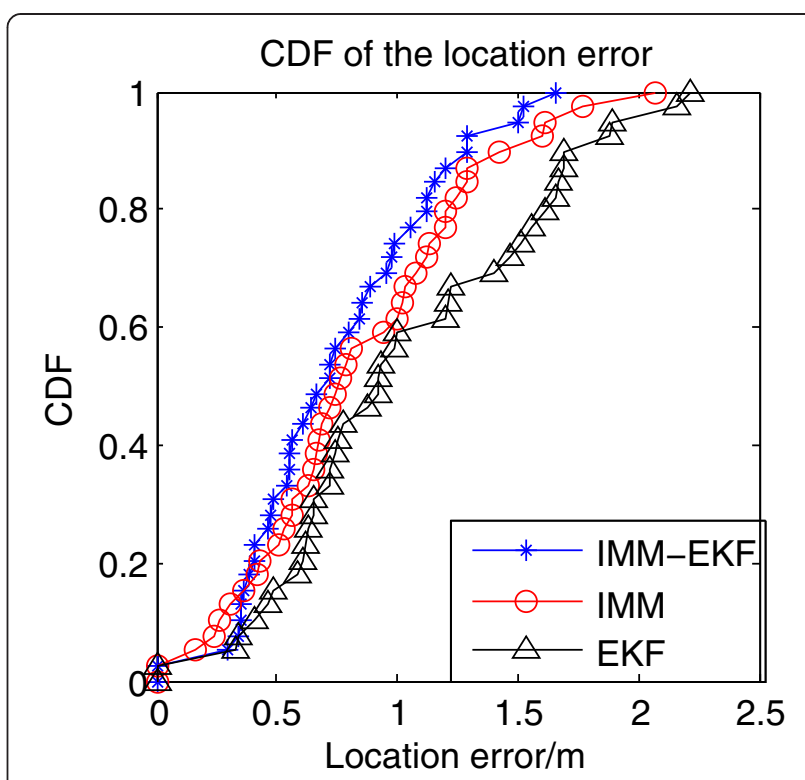

Figure $11 \mathrm{CDF}$ of the location error.

University. We adopt the NA5TR1 CSS nodes in the field experiments.

The experimental environment and deployment of the ANs are described in Figure 3. Five ANs are deployed on the ceiling within a $15 \mathrm{~m} \times 15 \mathrm{~m}$ area. $\mathrm{MN}$ is deployed at the height of $1.5 \mathrm{~m}$ above the floor. The coordinates of the five ANs are AN1 (8.59,6.85), AN2 (5.05,2.80), AN3 $(2.20,2.80)$, AN4 $(6.70,5.80)$, and AN5 $(10,10)$. The total length of the MN's moving path is $19 \mathrm{~m}$. The path lengths in office $1 \#$, the corridor, and office $3 \#$ are 8.5 , 6.5 , and $4 \mathrm{~m}$, respectively. The speed of $\mathrm{MN}$ is set as $M v=0.5 \mathrm{~m} / \mathrm{s}$. Sampling interval is set as $T i=1 \mathrm{~s}$. Figure 4 shows the propagation statistics between $\mathrm{MN}$ and ANs. The LOS/NLOS transition conditions between $\mathrm{MN}$ and [AN1, AN2, AN3, AN4] change into NLOS/LOS at time step 18, so do the conditions between $\mathrm{MN}$ and [AN1, AN4, AN5] at time step 31. The two-state Markov transition probability matrix is $p=\left[\begin{array}{lll}0.95 & 0.05 ; 0.05 & 0.95\end{array}\right]$. Since the initial probabilities of the both models are unknown, we assume them as $u=\left[\begin{array}{ll}0.5 & 0.5\end{array}\right]$. The initial input range estimations of the filters are set as $D(0)=$ $\left[d_{i}^{\text {mes }}(0) ; M v \times T i\right]$. Measurement noise parameters of LOS and NLOS conditions are obtained by off-line test, with variance of LOS error $R^{l o s}=0.5$, mean LOS error $\mu_{d}^{\text {los }}=0$, variance of NLOS error $R^{\text {nlos }}=4$, and NLOS

Table 2 Comparison of positioning error

\begin{tabular}{lllll}
\hline Algorithm & Maximum/m & Average/m & Deviation $/ \mathbf{m}$ & $\mathbf{9 0 \% m}$ \\
\hline IMM-EKF & 1.65 & 0.76 & 0.38 & 1.29 \\
IMM & 2.06 & 0.86 & 0.47 & 1.43 \\
EKF & 2.21 & 1.06 & 0.55 & 1.70 \\
\hline
\end{tabular}




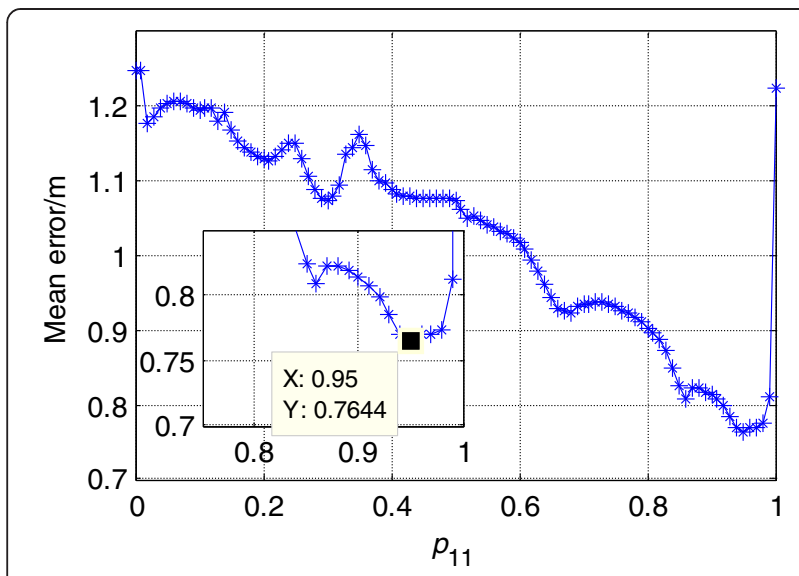

Figure 12 Sensitivity analysis of different Markov chain probabilities.

error mean $\mu_{d}^{\text {nlos }}=2.5$. To verify the effectiveness of the proposed IMM-EKF, we compare it with the IMM employed in [15] and the single model EKF localization algorithm. The IMM measurement filtering results are shown in Figure 7.

As the results of all ANs are similar, we just pick up AN1 as an example. Figure 7a shows the model probabilities of AN1. Contrast to the transition conditions of AN1, the probabilities of most of the time steps are reasonable. When AN1 is under the NLOS conditions, the NLOS model probability is larger than that of the LOS model, and when the condition turns into LOS, the probabilities will switch back timely. The detailed distance estimation errors along the path are shown in Figure 7b. It can be seen that at the time step $t$, the ranging errors are large due to the NLOS condition. However, with the IMM filtering, the errors are obviously decreased. The error statistics of all the ANs are shown in Table 1. The result demonstrates that the proposed IMM algorithm can effectively adapt the changes of the propagation conditions and significantly decrease the NLOS ranging errors.

With the filtered distance from the IMM algorithm, the MN's locations are calculated by the EKF. The IMM-EKF tracking result is compared with those of the IMM-only algorithm and EKF-only algorithm. Most of the related parameters in IMM are identical to IMMEKF. For comparison, the EKF algorithm adopts the measurement error distribution as Gaussian distribution $N(1,4)$. All visualized tracking results are shown in Figures 8,9 , and 10 . The positioning errors are indicated by the lines connecting the estimated location (*) and real location (dots). The cumulative distribution function (CDF) of positioning error is shown in Figure 11.

As a valid algorithm for switching mode, the IMM algorithm has only been used successfully for LOS and NLOS environments in urban area. However, for wireless signal transmission, there is a large difference between outdoor and indoor environments. To compare the performance and effects of the different methods, the algorithm proposed in [15] is modified at some extent to be used for comparison in indoor LOS/NLOS environment. From the simulation results shown in Figures 8, 9, 10, and 11, the proposed IMM-EKF smoother can more accurately track the true range distances in indoor LOS/NLOS mixed situations. The main reason lies in that the mean noise error is introduced into the weighted fusion estimation at the stage of ranging filter, as shown in Equation (26). At the same time, the EKF algorithm goes a step further to depress the localization error. From the CDF of the positioning error in Figure 11, we can intuitively see the error distribution of EKF, IMM, and IMM-EKF algorithms. When the EKF and IMM are employed in the LOS/NLOS mixed environment, the localization procedure can achieve the accuracy of about $2.3 \mathrm{~m}$. However, the IMM-EKF can achieve higher accuracy with $100 \%$ location error below $1.7 \mathrm{~m}$, which is the best. Above all, the single model cannot fit the LOS/NLOS mixed environment very well.

The detailed statistical characters about the maximum, average, standard deviation, and $90 \%$ of the positioning error are summarized in Table 2. With the IMM-EKF algorithm, we can expect a localization performance of $90 \%$ error below $1.29 \mathrm{~m}$. For general applications such as personal tracking and robot navigation, it is an acceptable result.

Experiments with different Markov chain probabilities are carried out to investigate the relationship between the Markov chain probabilities and localization performance. We assume that $p_{11}+p_{12}=1$ and $p_{12}=$ $p_{21}$. Therefore, once one of the transition probabilities is determined, the others are also fixed. So, we select $p_{11}$ as the variable to conduct the evaluation. Figure 12 shows the variation of the mean positioning errors due to the alteration of Markov chain probability. When the $p_{11}=$ 0.95 , the IMM-EKF achieves the best localization accuracy.

\section{Conclusions}

This paper proposes a real-time localization algorithm, based on IMM-EKF, for moving target localization under LOS/NLOS mixed indoor environment. When the system obtains the measured distance vector between the $\mathrm{MN}$ and the ANs, the IMM is employed to mitigate the ranging errors either for the LOS or NLOS conditions.

With the filtered ranging result, the EKF algorithm is adopted to estimate the position of MN. In the range filtering stage, the measurement errors, especially the NLOS errors, are obviously weakened. By prediction and update, the EKF-based localization stage makes further improvement on the accuracy. Experimental result shows that the algorithm proposed in this paper achieves high accuracy even in complex environment where 
LOS and NLOS channel conditions switch randomly and frequently. This research is accomplished on the basis of a priori knowledge of the ranging error distribution for LOS and NLOS conditions. For further works, we will focus on the research to update the error parameters during the localization process.

\section{Abbreviations}

AN: Anchor node; AOA: Angle of arrival; CDF: Cumulative distribution function; CSS: Chirp spread spectrum; EKF: Extended Kalman filter; GPS: Global position system; i.i.d: Independently and identically distributed; IMM: Interacting multiple model; KF: Kalman filter; LP: Linear programming; LOS: Line-of-sight; MN: Moving node; NLOS: Non-line of sight; RSS: Receive signal strength; Rwgh: Residual weighting; SDS-TWR: Symmetric double-sided two-way ranging; TN: Target node; TOA: Time of arrive; TOF: Time of flight; UWB: Ultra wideband; WSN: Wireless sensor networks.

\section{Competing interests}

The authors declare that they have no competing interests.

\section{Acknowledgements}

This research was supported by the National Natural Science Foundation of China (grant no. 61273078), China Postdoctoral Science Foundation (grant no. 2012 M511164), Chinese Universities Scientific Foundation (grant nos. N1 10404030 and N110404004), Liaoning Doctoral Foundation (grant no. 20121004)

Received: 22 July 2013 Accepted: 10 December 2013

Published: 28 December 2013

\section{References}

1. ED Kaplan, CJ Hegarty, Understanding GPS: Principles and Applications (Artech House, Boston, 2005)

2. J Khodjaev, Y Park, AS Malik, Survey of NLOS identification and error mitigation problems in UWB-based positioning algorithms for dense environments. Ann. Telecommun. 65, 301-311 (2010)

3. S Venkatesh, RM Buehrer, A linear programming approach to NLOS error mitigation in sensor networks, in Proceedings of the 5th International Conference on Information Processing in Sensor Networks (Nashville, TN, USA, 2006), pp. 301-308

4. $\quad Y$ Geng, $\mathrm{H}$ Deng, Modeling the effect of human body on TOA ranging for indoor human tracking with wrist mounted sensor, in 2013 16th International Symposium on Wireless Personal Multimedia Communications (WPMC) (Atlantic City, NJ, USA, 2013), pp. 1-6

5. JE Kim, J Kang, D Kim, Y Ko, J Kim, IEEE 802.15.4a CSS-based localization system for wireless sensor networks, in IEEE International Conference on Mobile Adhoc and Sensor Systems (MASS 2007) (Pisa, 2007), pp. 1-3

6. A Sikora, VF Groza, Fields tests for ranging and localization with time-of-flightmeasurements using chirp spread spectrum RF-devices, in 2007 IEEE Instrumentation and Measurement Technology Conference (, Warsaw, 2007), pp. 1-6

7. G Destino, D Macagnano, GTF de Abreu, Hypothesis testing and iterative WLS minimization for WSN localization under LOS/NLOS conditions, in Conference Record of the Forty-First Asilomar Conference on Signals, Systems and Computers (Pacific Grove, CA, USA, 2007), pp. 2150-2155

8. G Ismail, C Chia-Chin, W Fujio, I Hiroshi, NLOS identification and weighted least-squares localization for UWB systems using multipath channel statistics. EURASIP J. Adv. Signal Process. 2008, 271984 (2008)

9. KT Feng, CL Chen, CH Chen, GALE: an enhanced geometry-assisted location estimation algorithm for NLOS environments. IEEE Trans. Mob. Comput 7, 199-213 (2008)

10. Y Kong, Y Kwon, G Park, Robust localization over obstructed interferences for inbuilding wireless applications. IEEE Trans. Consum. Electron. 55, 105-111 (2009)

11. S Marano, WM Gifford, H Wymeersch, MZ Win, NLOS identification and mitigation for localization based on UWB experimental data. IEEE J. Selected Areas Commun. 28, 1026-1035 (2010)

12. SH Fang, N Lin, A dynamic system approach for radio location fingerprinting in wireless local area networks. IEEE Trans. Commun $58,1020-1025$ (2010)
13. J Wang, Q Gao, Y Yu, H Wang, M Jin, Toward robust indoor localization based on Bayesian filter using chirp-spread-spectrum ranging. IEEE Trans. Ind. Electron. 59, 1622-1629 (2012)

14. JF Liao, BS Chen, Robust mobile location estimator with NLOS mitigation using interacting multiple model algorithm. IEEE Trans. Wirel. Commun 5, 3002-3006 (2006)

15. BS Chen, CY Yang, FK Liao, JF Liao, Mobile location estimator in a rough wireless environment using extended Kalman-based IMM and data fusion. IEEE Trans. Veh. Technol 58, 1157-1169 (2009)

16. BS Choi, JW Lee, JJ Lee, KT Park, A hierarchical algorithm for indoor mobile robot localization using RFID sensor fusion. IEEE Trans. Ind. Electron 58, 2226-2235 (2011)

17. H Guo, KS Low, HA Nguyen, Optimizing the localization of a wireless sensor network in real time based on a low-cost microcontroller. IEEE Trans. Ind. Electron. 58, 741-749 (2011)

18. W Xu, Z Wang, SR Zekavat, Non-line-of-sight identification via phase difference statistics across two-antenna elements. IET Commun. 5, 1814-1822 (2011)

19. X Lin, L Ju, S Chen, NLOS error identification and range approximation technique in cellular networks, in 4th International Conference on Wireless Communications, Networking and Mobile Computing (Dalian, Dalian, 2008), pp. 1-4

20. S Venkatesh, R Buehrer, Non-line-of-sight identification in ultra-wideband systems based on received signal statistics. IET Microw. Antenna. P. 1, 1120-1130 (2007)

21. M Heidari, NA Alsindi, K Pahlavan, UDP identification and error mitigation in TOA-based indoor localization systems using neural network architecture. IEEE Trans. Wirel. Commun. 8, 3597-3607 (2009)

22. M Heidari, F Akgul, K Pahlavan, Identification of the absence of direct path in indoor localization systems, in IEEE 18th International Symposium on Personal, Indoor and Mobile Radio Communications (Athens, 2007), pp. 1-6

23. YT Chan, WY Tsui, HC So, P Ching, Time-of-arrival based localization under NLOS conditions. IEEE Trans. Veh. Technol 55, 17-24 (2006)

24. S Marano, WM Gifford, H Wymeersch, MZ Win, Nonparametric obstruction detection for UWB localization, in IEEE Global Telecommunications Conference (Honolulu, HI, USA, 2009), pp. 1-6

25. S Venkatesh, RM Buehrer, NLOS mitigation using linear programming in ultrawideband location-aware networks. IEEE Trans. Veh. Technol. 56, 3182-3198 (2007)

26. $\mathrm{PC}$ Chen, A non-line-of-sight error mitigation algorithm in location estimation. WCNC 1, 316-320 (1999)

27. PC Chen, A cellular based mobile location tracking system. VETEC 3, 1979-1983 (1999)

28. U Hammes, AM Zoubir, Robust mobile terminal tracking in NLOS environments based on data association. IEEE Trans. Signal Process. 58, 5872-5882 (2010)

29. K Yu, E Dutkiewicz, Geometry and motion-based positioning algorithms for mobile tracking in NLOS environments. IEEE Trans. Mob. Comput. 11, 254-263 (2012)

30. S Nawaz, N Trigoni, Robust localization in cluttered environments with NLOS propagation, in IEEE 7th International Conference on Mobile Adhoc and Sensor Systems (MASS) (San Francisco, CA, USA, 2010), pp. 166-175

31. L Jiao, JP Xing, J Zhang, X Zhang, ZL Zhao, A new NLOSTOA-based wireless sensor network localization algorithm with robust character. Chin. J. Sensors Actuators 20, 1625-1629 (2007)

32. H Wymeersch, S Maranò, WM Gifford, MZ Win, A machine learning approach to ranging error mitigation for UWB localization. IEEE Trans. Commun. 60, 1719-1728 (2012)

33. C-Y Yang, B-S Chen, F-K Liao, Mobile location estimation using fuzzy-based IMM and data fusion. IEEE Trans. Mob. Comput 9, 1424-1436 (2010)

34. Y Jiang, VCM Leung, IEEE International Symposium on Signals, Systems and Electronics (Montreal, Quebec, 2007), pp. 525-528

\section{doi:10.1186/1687-1499-2013-291}

Cite this article as: Zhang et al:: Moving target localization in indoor wireless sensor networks mixed with LOS/NLOS situations. EURASIP Journal on Wireless Communications and Networking 2013 2013:291. 